\title{
Risk stratification for lung nodules: Size isn't everything
}

\author{
Takashi Eguchi, MD, PhD, and Prasad S. Adusumilli, MD, FACS, FCCP
}

\footnotetext{
From the Thoracic Service, Department of Surgery, Memorial Sloan Kettering Cancer Center, New York, NY. The author's laboratory is supported by grants from the National Institutes of Health (P30 CA008748 and R21 CA164568-01A1), U.S. Department of Defense (LC110202 and LC160212), and the Mr William H. Goodwin and Alice Goodwin, the Commonwealth Foundation for Cancer Research, and the Experimental Therapeutics Center.

Received for publication Oct 27, 2016; revisions received Nov 28, 2016; accepted for publication Dec 17, 2016; available ahead of print March 14, 2017

Address for reprints: Prasad S. Adusumilli, MD, FACS, FCCP, Thoracic Service, Department of Surgery, Member, Centers for Cell Engineering and Experimental Therapeutics and Cellular Therapeutics, Memorial Sloan Kettering Cancer Center, 1275 York Ave, New York, NY 10065 (E-mail: adusumip@mskcc.org).

J Thorac Cardiovasc Surg 2017;153:1557-62 $0022-5223 / \$ 36.00$

Copyright (C) 2017 by The American Association for Thoracic Surgery http://dx.doi.org/10.1016/j.jtcvs.2016.12.068
}

With the demonstration of the efficacy of low-dose computed tomography (CT) screenings for lung cancer in the National Lung Screening Trial, the detection of earlystage lung cancer is expected to increase. ${ }^{1}$ Small lung cancers $\leq 2 \mathrm{~cm}$ (T1a in the seventh edition of the tumor, node, and metastasis $[\mathrm{TNM}]$ classification) have been categorized further into T1a $(\leq 1 \mathrm{~cm})$ and T1b ( $>1$ to $\leq 2 \mathrm{~cm})$ in the upcoming eighth edition. ${ }^{2}$ This change was based on prognostic data from a multinational cohort of the International Association for the Study of Lung Cancer (IASLC), which is considered the reference standard for overall survival for patients with non-small cell lung cancer (NSCLC). The TNM classification is a simple and clinically useful prognosticator. Increasing patient age, however, is associated with more comorbidities and high competing risks $^{3}$; therefore, the consideration of multiple available treatment options (sublobar vs lobar resection, or stereotactic ablative radiotherapy) and risk stratification beyond tumor size are required in the management of both solitary lung nodules and early-stage lung cancers.

More than $80 \%$ of all lung cancers are NSCLC, among which adenocarcinoma (ADC) is the most common histologic subtype, and its incidence is increasing. In an effort to better prognosticate lung ADC beyond the use of the T component of the TNM staging classification, a multidisciplinary group composed of experts from the IASLC, American Thoracic Society (ATS), and European Respiratory Society (ERS) proposed a new classification. The foundation of this new classification was the evidence that histologic subtype can predict tumor behavior and malignancy. ${ }^{4}$ The World Health Organization adopted this system in 2015, after validation in independent cohorts.

In this classification, 4 major concepts were recommended: (1) histologic patterns are recorded in 5\% increments, and the predominant histologic pattern subsequently is determined (lepidic [LEP], acinar, papillary, micropapillary [MIP], and solid [SOL]); (2) MIP pattern was added as a major histologic subtype, owing to its association with poor

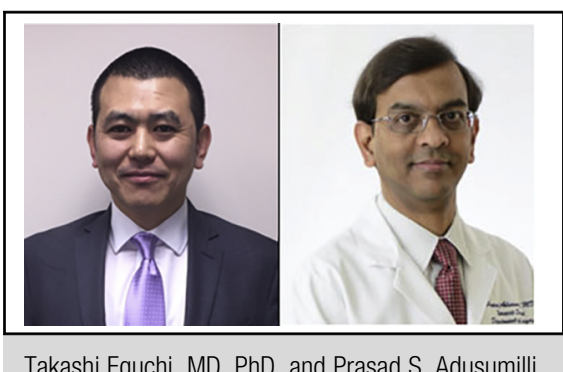

Takashi Eguchi, MD, PhD, and Prasad S. Adusumilli, MD, FACS, FCCP

\section{Central Message}

Beyond tumor size, the presence of high-risk features on radiologic imaging, preoperative biopsy, and postoperative histologic subtype should be considered in the risk stratification of small lung nodules.

See Editorial Commentary page 1563.

prognosis; (3) 2 new subtype categories were added, adenocarcinoma in situ (AIS) and minimally invasive adenocarcinoma (MIA); and (4) mucinous bronchioloalveolar carcinoma was reclassified as invasive mucinous adenocarcinoma (IMA). This histologic classification had implications for the Fleischner Society recommendations for the management of sub-solid pulmonary nodules detected by CT scan. ${ }^{6}$ Additionally, the IASLC Lung Cancer Staging Project addressed the correlation between radiologic partsolid nodules and the histologic components of ADC by proposing the revised $\mathrm{T}$ categories and tumor size assessment in the eighth edition of the TNM classification.

Herein, to address the pressing need to manage small lung nodules detected on CT scans, we summarize the published evidence and discuss key issues related to diagnostic and staging workup, choice of resection type (lobectomy vs sublobar resection), and postoperative risk assessment. After the recorded decrease in cigarette smoking, the incidence of squamous cell carcinoma has declined. Squamous cell carcinoma accounts for $<20 \%$ of all early-stage NSCLC tumors ${ }^{3}$; therefore, the article is focused primarily on the management of lung ADC.

\section{AT PRESENTATION OF A LUNG NODULE: FURTHER WORKUP OR FOLLOW-UP?}

Pure ground-glass nodules identified on first presentation are followed with further imaging. ${ }^{6}$ Solid and sub-solid nodules detected by CT scans require further work-up. The Fleischner Society and, ${ }^{6}$ after considering new data from the National Lung Screening Trial and other trials, the National 


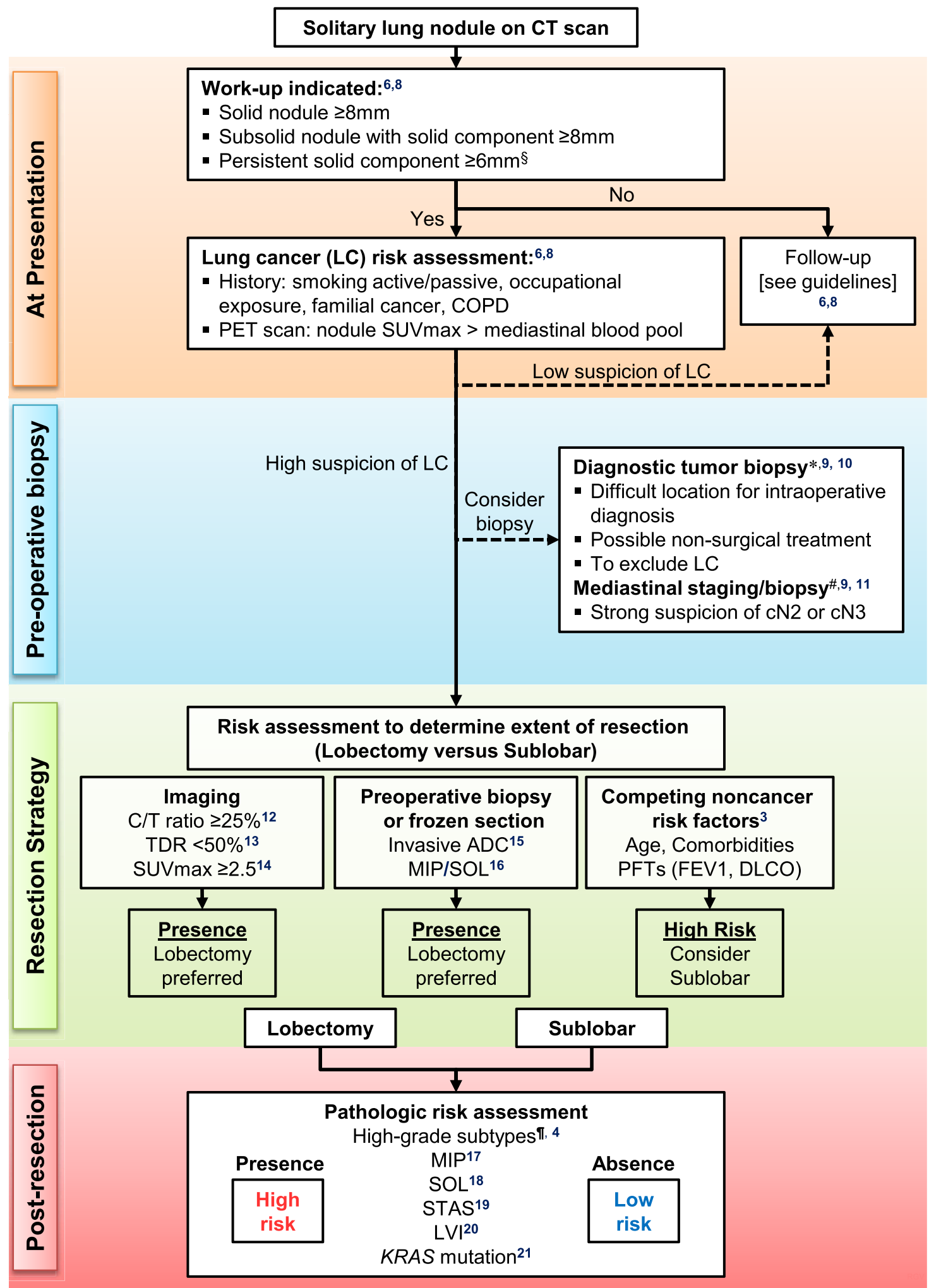

FIGURE 1. Risk stratification for small nodules. ${ }^{3,4,6,8-21} \oint$ Persistent solid component $\geq 6 \mathrm{~mm}$ in the follow-up CT scan. *Adequate tissue is needed for both histologic and molecular testing. \#EBUS is the preferred first choice on the basis of invasiveness, sensitivity, and the relative cost of mediastinoscopy. \High-grade subtypes: MIP, SOL, or colloid-predominant subtypes, or invasive mucinous adenocarcinoma. $C T$, Computed tomography; $L C$, lung cancer; $C O P D$, chronic obstructive pulmonary disease; PET, positron emission tomography; SUVmax, maximum standardized uptake value; Sublobar, sublobar resection; $C /$ T ratio, consolidation to tumor ratio; $A D C$, adenocarcinoma; $T D R$, tumor disappearance ratio; $M I P$, micropapillary; $S O L$, solid; $P F T$, pulmonary function test; FEV1, forced expiratory volume in the first second; $D L C O$, diffusion capacity of the lungs for carbon monoxide; STAS, spread through air spaces; $L V I$, lymphovascular invasion. 
Comprehensive Cancer Network recently published guidelines for lung cancer screening and workup of lung nodules. ${ }^{8}$ On the basis of these guidelines and other published evidence, we propose an algorithmic management of CT-detected solitary lung nodules (Figure 1). , $^{3,6,8-21}$

Initial diagnostic work-up with positron emission tomography/CT should be considered for any nodule that has a solid component $\geq 8 \mathrm{~mm}$ or for sub-solid nodules with a persistent solid component $\geq 6 \mathrm{~mm} .{ }^{8}$ The results of this imaging-along with patient risk factors for lung cancer (eg, passive/active smoking, occupational exposure, smokingrelated other cancer history, family history, and chronic obstructive pulmonary disease) - are used to determine whether the nodule is highly suspicious for lung cancer, which would necessitate further workup/treatment, or not suspicious for lung cancer, which would allow for management with close follow-up only. ${ }^{6}$

\section{TO PERFORM THE PREOPERATIVE BIOPSY OR NOT? Preoperative Diagnostic Biopsy}

Patients for whom there is strong clinical and radiologic suspicion of stage I disease, on the basis of lung cancer risk assessment, may not require a diagnostic biopsy before resection, unless intraoperative diagnosis appears to be difficult or risky ${ }^{9}$ and/or preoperative histologic information influences the type of surgical resection. Because of the therapeutic implications of the recent advances in histologic subtyping, diagnostic core-needle biopsy ${ }^{22}$ is substantive enough to allow for both histologic and molecular testing. ${ }^{23}$ Patients for whom there is suspicion of lung cancer and who are being considered for nonsurgical therapy require histologic confirmation before initiation of treatment. ${ }^{9}$

In the ongoing multicenter Cancer and Leukemia Group B (CALGB) 140503 trial, Kohman and colleagues ${ }^{10}$ investigated the factors preventing the intraoperative randomization of patients with clinical stage IA NSCLC $\leq 2 \mathrm{~cm}$ to be treated with lobectomy or sublobar resection. Of 637 preregistered patients, only $61 \%$ were randomized. Of the nonrandomized patients $(39 \%),>80 \%$ were found to be misdiagnosed (benign nodules) or understaged. The reasons for nonrandomization ( $\mathrm{n}=208$ ) were (1) NSCLC not confirmed (benign or other malignancy) by intraoperative frozen-section analysis (58\%); (2) NSCLC confirmed but patient ineligible because of advanced stage $(23 \%)$; (3) technical reasons $(6 \%)$; and (4) other reasons $(14 \%)$. There was a statistically significant increase in successful randomization (50\% vs $23 \%$ ) among patients who had preoperative diagnostic biopsies. This study concluded that preoperative biopsy for radiologically suspected small NSCLC would reduce the number of nontherapeutic or unnecessary thoracic procedures.

\section{Preoperative Invasive Mediastinal Staging}

Intraoperative mediastinal node staging is recommended before planned resection (during the same anesthetic event) for all patients diagnosed with clinical stage I or II lung cancer, except those with radiologically solid tumors $<1 \mathrm{~cm}$ or pure nonsolid tumors $\leq 3 \mathrm{~cm}$, because of the low likelihood of positive mediastinal lymph nodes. ${ }^{9}$ Preoperative staging is recommended when there is strong clinical suspicion of $\mathrm{N} 2$ or N3 nodal disease. The utility of endobronchial ultrasound-guided transbronchial needle aspiration (EBUS-TBNA) and/or mediastinoscopy in detecting occult nodal metastasis in clinical stage I lung cancer is an ongoing focus of investigation. ${ }^{24}$

Czarnecka-Kujawa and colleagues ${ }^{11}$ conducted a retrospective, cost-effectiveness analysis of mediastinal lymph node staging in NSCLC and compared 4 strategies on the basis of cost, sensitivity, and risk of $\mathrm{pN} 2$ in clinical N0 patients: (1) no invasive staging before surgery; (2) EBUS-TBNA; (3) mediastinoscopy; and (4) EBUSTBNA followed by confirmatory mediastinoscopy. This study concluded that, in patients with low pN2 risk $(<2.5 \%)$ invasive mediastinal staging was not cost-effective, in moderate-risk patients $(2.5 \%-57 \%)$ EBUS-TBNA was cost-effective when used as the only staging modality, and in high-risk patients $(>57 \%)$ confirmatory mediastinoscopy should be considered in cases of negative EBUS-TBNA.

We reported previously that the histologic presence of MIP pattern and the absence of LEP pattern were independent predictors of occult mediastinal lymph node metastasis in patients with clinical N0-1 lung ADC (tumor size $\leq 5 \mathrm{~cm}){ }^{25}$ In this consecutive case study cohort, $11 \%$ of all patients had occult $\mathrm{N} 2$ disease and the risk of occult $\mathrm{N} 2$ disease increased as the percentage of MIP pattern increased (MIP pattern: $<5 \%, 5 \%-39 \%$, and $\geq 40 \%$; occult N2: $6 \%, 13 \%$, and $26 \%$, respectively). The presence of SOL pattern also was associated with a high risk of occult N2 disease. According to the risk-specific mediastinal staging strategy reported by Czarnecka-Kujawa and colleagues, ${ }^{11}$ if presence of MIP and/or SOL pattern is recognized on preoperative tumor biopsy specimens, preoperative EBUS-TBNA would be appropriate on the basis of the high risk of positive $\mathrm{N} 2$ nodes. A combination of high-risk clinical and radiologic features, together with the presence of MIP and/or SOL histologic pattern on preoperative biopsy, necessitates preoperative EBUS-TBNA.

\section{RESECTION TYPE: LOBECTOMY OR SUBLOBAR?}

The standard treatment for stage I NSCLC is lobectomy with mediastinal lymph node evaluation. ${ }^{26}$ An analysis of Surveillance, Epidemiology, and End Results data between 2001 and 2007, however, demonstrated that, among elderly patients ( $\geq 66$ years of age), only $59 \%$ had undergone lobectomy, with this percentage decreasing as age increased. ${ }^{27}$ Despite concerns about the adequacy of sublobar resection as a curative option, ${ }^{28}$ its use has been increasing. Sublobar resection (segmentectomy and wedge 
resection) was considered an alternative treatment for (1) elderly patients $\geq 75$ years of age ${ }^{26}$; (2) patients with poor pulmonary reserve or other major comorbidities that contraindicate lobectomy ${ }^{9}$; and (3) patients with peripheral nodules $\leq 2 \mathrm{~cm}$ with pure AIS histologic pattern, $\geq 50 \%$ ground-glass appearance on CT scan, or surveillanceconfirmed long doubling time ( $\geq 400$ days). ${ }^{9}$ Data from ongoing randomized clinical trials (CALGB 140503 and Japan Clinical Oncology Group [JCOG] 0802) assessing the outcomes of sublobar versus lobar resection of small $(\leq 2 \mathrm{~cm})$ tumors can add to the accuracy of individual lung cancer-specific prognostic risk assessment.

\section{Preoperative Radiologic Risk Assessment}

JCOG 0201 prospectively investigated the utility of thinsection CT to predict pathologically noninvasive tumors. In 545 radiology and pathology evaluations, the use of 2 radiologic criteria-consolidation/tumor $(\mathrm{C} / \mathrm{T})$ ratio $\leq 0.25$ in lung $\mathrm{ADC}$ tumors $\leq 2 \mathrm{~cm}$ and $\mathrm{C} / \mathrm{T}$ ratio $\leq 0.5$ in lung $\mathrm{ADC}$ tumors 2 to $3 \mathrm{~cm}$-was able to predict excellent prognosis in patients after lobectomy. ${ }^{12} \mathrm{~A}$ separate multi-institution retrospective study showed that $\mathrm{C} / \mathrm{T}$ ratio $\leq 0.25$ was associated with excellent prognosis in lobectomy-tolerant patients ( $\leq 75$ years of age) who had undergone sublobar resection, thus suggesting that tumors with a $\mathrm{C} / \mathrm{T}$ ratio $\leq 0.25$ are good candidates for sublobar resection. ${ }^{29}$ Shimada and colleagues ${ }^{13}$ investigated the predictive value of radiologic tumor disappearance ratio for pathologic invasiveness in cT1a $(\leq 2 \mathrm{~cm})$ NOM0 NSCLC. The prognostic value of tumor disappearance ratio was validated subsequently in a prospective trial.

Okada and colleagues ${ }^{14}$ investigated the prognostic predictive value of maximum standardized uptake value (SUVmax) from positron emission tomography scans and reported that, in addition to CT findings, SUVmax (optimal cutoff $\geq 2.5$ ) was useful in predicting high-grade pathologic features (lymphovascular/pleural invasion and lymph node metastasis) and worse prognosis after lung resection in patients with clinically diagnosed T1N0M0 lung ADC. Our group reported that C/T ratio of 0.25 and SUVmax of 2.2 (median of the cohort) were useful markers in stratifying the risk of recurrence after sublobar resection in patients with cT1a $(\leq 2 \mathrm{~cm})$ NOM0 lung ADC. ${ }^{30}$ Nakamura and colleagues $^{31}$ reported a close association between SUVmax and predominant histologic subtypes based on the new classification - the highest SUVmax was observed in MIP, followed by SOL, IMA, acinar, papillary, LEP, MIA, and AIS.

\section{Risk Assessment Based on Preoperative Biopsy or Intraoperative Frozen-Section Analysis}

Histologic subtyping using cytologic specimens is challenging. Ferretti and colleagues ${ }^{32}$ reported that CT-guided transthoracic core-needle biopsy specimens would be useful for both histologic subtyping and molecular testing for lung ADC in accordance with the new IASLC/American Thoracic Society (ATS)/European Respiratory Society (ERS) classification system. Liu and colleagues ${ }^{15}$ conducted a retrospective investigation of the ability of intraoperative frozen-section analysis to distinguish AIS and MIA versus invasive $A D C$ in patients with peripheral small lung ADC. They reported high concordance with the final pathologic assessment $(84 \%)$. Because invasive ADC includes different histologic predominant subtypes of variable prognostic significance, we investigated the utility of frozen-section analysis in predicting each specific histologic subtype and found that, for detecting the presence of MIP and SOL patterns, frozen-section analysis has high specificity ( $94 \%$ and $96 \%$, respectively) but low sensitivity (37\% and $69 \%$, respectively). ${ }^{16}$

\section{Competing Noncancer Risk Assessment}

Among solid tumors, lung cancer carries a relatively high risk of competing cancer and noncancer events because more than two-thirds of patients with lung cancer are $\geq 65$ years of age at the time of diagnosis, one half of whom are $\geq 75$ years of age. ${ }^{3}$ Among elderly patients, particularly those with early-stage disease, overall survival, disease-free survival, and recurrence-free survival are affected by competing events, which underlines the need to perform competing risks analysis during preoperative assessment. ${ }^{3}$ As age increases, the risk of competing events, such as death from noncancer diseases, also increases. Noncancer risk factors for poor outcomes include increased age, comorbidities (such as chronic obstructive pulmonary disease and cardiovascular disease), and poor pulmonary function, as determined by forced expiratory volume in the first second and diffusion capacity of the lungs for carbon monoxide.

\section{POSTRESECTION PATHOLOGIC RISK ASSESSMENT}

The new classification of lung ADC recommends that pathologists report predominant subtypes in 5\% increments for all subtypes. Colloid-predominant, MIP, SOL, and IMA tumors were classified as high-grade on the basis of clinicopathologic analysis of patients with stage I lung $\mathrm{ADC}$ and were validated subsequently in separate international cohorts.

In addition to predominant subtype, the presence of any high-grade subtypes, such as MIP or SOL, has been reported to be associated with worse prognosis after lung resection. ${ }^{17,18}$ We investigated the procedure-specific prognostic impact of presence of MIP $(\geq 5 \%)$ in small $(\leq 2 \mathrm{~cm})$ lung $\mathrm{ADC}$ and found that presence of MIP was associated with a greater incidence of recurrence in patients who had undergone sublobar resection but not in those who had undergone lobectomy. ${ }^{17}$ 
Other than histologic subtype, pathologic prognosticators include tumor spread through air spaces (STAS), which we defined as tumor cells within alveolar spaces in the lung parenchyma beyond the edge of the main tumor. This is a recently recognized pattern of tumor invasion in lung cancers and has been reported to be associated with worse prognosis in patients after lung resection. ${ }^{19}$ We reported the procedure-specific prognostic impact of tumor STAS in patients with small $(\leq 2 \mathrm{~cm})$ lung ADC-the poor prognostic influence of STAS was more significant in patients who had undergone sublobar resection. ${ }^{33} \mathrm{We}$ also recently reported the incidence of tumor STAS in lung squamous cell carcinoma and its prognostic impact on recurrence and lung cancer-specific survival in a competing risks analysis. ${ }^{34}$ Additionally, microscopic lymphovascular invasion has been shown to be associated with worse prognosis, and cellular findings, such as mitotic count, are considered useful prognosticators in resected lung cancer. ${ }^{20,35}$ Finally, we reported that KRAS mutation in early-stage lung ADC was a strong predictor of greater recurrence and worse survival, especially for patients with SOL-predominant tumors. ${ }^{21}$ Risk assessment based on molecular testing also can be performed preoperatively with biopsy specimens. ${ }^{36}$

Aside from the aforementioned strategies, molecularbased risk assessments, such as cell cycle progression score $^{36}$ and immune microenvironment-based risk assessments (tumor-infiltrated $\mathrm{T}$ cells, tumor-associated Tregs, and tumoral cytokine receptor expression), ${ }^{37}$ have been reported to be useful in risk assessment; their utility and integration in clinical practice have yet to be validated. Although the significance of emerging biomarkers in the therapeutic management of high-risk, early-stage lung ADC remains undefined, these patients should be reviewed in a multidisciplinary setting for potential benefits of additional therapies.

\section{Summary}

The high rate of misdiagnosis and/or understaging of solitary small lung nodules despite best management practices, combined with the high rates of recurrence seen in a subgroup of early-stage patients undergoing curative-intent resection, necessitates risk stratification beyond tumor size in the management of small-sized lung nodules. Our algorithmic summarization of the published evidence can help identify and stratify subgroups of patients who have a greater risk of recurrence or death. Furthermore, it can assist in individualizing treatment plans that include different treatment modalities (surgery or stereotactic ablative radiotherapy), different surgical procedures (lobectomy or sublobar resection), and additional postsurgical therapies (completion lobectomy, adjuvant chemotherapy, or observation).

\section{Conflict of Interest Statement}

Authors have nothing to disclose with regard to commercial support.

We thank David Sewell and Alex Torres of the Memorial Sloan Kettering Thoracic Surgery Service for their editorial assistance.

\section{References}

1. Aberle DR, Adams AM, Berg CD, Black WC, Clapp JD, Fagerstrom RM, et al Reduced lung-cancer mortality with low-dose computed tomographic screening. N Engl J Med. 2011;365:395-409.

2. Goldstraw P, Chansky K, Crowley J, Rami-Porta R, Asamura H, Eberhardt WE, et al. The IASLC Lung Cancer Staging Project: Proposals for Revision of the TNM Stage Groupings in the Forthcoming (Eighth) Edition of the TNM Classification for Lung Cancer. J Thorac Oncol. 2016;11:39-51.

3. Eguchi T, Bains S, Lee M, Tan KS, Hristov B, Buitrago DH, et al. Impact of increasing age on cause-specific mortality and morbidity in patients with stage I non-small-cell lung cancer: a competing risks analysis. J Clin Oncol. 2017 $35: 281-90$.

4. Travis WD, Brambilla E, Noguchi M, Nicholson AG, Geisinger KR, Yatabe Y, et al. International association for the study of lung cancer/american thoracic society/european respiratory society international multidisciplinary classification of lung adenocarcinoma. J Thorac Oncol. 2011;6:244-85.

5. Travis WD, Brambilla E, Nicholson AG, Yatabe Y, Austin JH, Beasley MB, et al. The 2015 World Health Organization Classification of Lung Tumors: Impact of Genetic, Clinical and Radiologic Advances Since the 2004 Classification. J Thorac Oncol. 2015;10:1243-60.

6. Naidich DP, Bankier AA, MacMahon H, Schaefer-Prokop CM, Pistolesi M, Goo JM, et al. Recommendations for the management of subsolid pulmonary nodules detected at CT: a statement from the Fleischner Society. Radiology. 2013;266:304-17.

7. Travis WD, Asamura H, Bankier AA, Beasley MB, Detterbeck F, Flieder DB, et al. The IASLC Lung Cancer Staging Project: Proposals for Coding T Categories for Subsolid Nodules and Assessment of Tumor Size in Part-Solid Tumors in the Forthcoming Eighth Edition of the TNM Classification of Lung Cancer. $J$ Thorac Oncol. 2016;11:1204-23.

8. National Comprehensive Cancer Centers. NCCN clinical practice guidelines in oncology (NCCN Guidelines): Lung cancer screening v1. 2017. Available at: http://www.nccn.org/professionals/physician_gls/pdf/lung_screening.pdf. Accessed October 20, 2016.

9. National Comprehensive Cancer Centers. NCCN clinical practice guidelines in oncology (NCCN Guidelines): Non-small cell lung cancer v4. 2016. Available at: http://www.nccn.org/professionals/physician_gls/pdf/nscl.pdf. Accessed October 20, 2016.

10. Kohman LJ, Gu L, Altorki N, Scalzetti E, Veit LJ, Wallen JM, et al. Biopsy first: lessons learned from CALGB 140503. J Thorac Cardiovasc Surg. 2017;153: 1592-7.

11. Czarnecka-Kujawa K, Rochau U, Siebert U, Atenafu E, Darling G, Waddell TK, et al. Cost-effectiveness of mediastinal lymph node staging in non-small cell lung cancer. J Thorac Cardiovasc Surg. 2017;153:1567-78.

12. Asamura H, Hishida T, Suzuki K, Koike T, Nakamura K, Kusumoto M, et al Radiographically determined noninvasive adenocarcinoma of the lung: survival outcomes of Japan Clinical Oncology Group 0201. J Thorac Cardiovasc Surg. 2013;146:24-30.

13. Shimada Y, Yoshida J, Hishida T, Nishimura M, Ishii G, Nagai K. Predictive factors of pathologically proven noninvasive tumor characteristics in T1aNOM0 peripheral non-small cell lung cancer. Chest. 2012;141:1003-9.

14. Okada M, Nakayama H, Okumura S, Daisaki H, Adachi S, Yoshimura M, et al Multicenter analysis of high-resolution computed tomography and positron emission tomography/computed tomography findings to choose therapeutic strategies for clinical stage IA lung adenocarcinoma. J Thorac Cardiovasc Surg. 2011;141:1384-91.

15. Liu S, Wang R, Zhang Y, Li Y, Cheng C, Pan Y, et al. Precise diagnosis of intraoperative frozen section is an effective method to guide resection strategy for peripheral small-sized lung adenocarcinoma. J Clin Oncol. 2016;34: 307-13.

16. Yeh YC, Nitadori J, Kadota K, Yoshizawa A, Rekhtman N, Moreira AL, et al. Using frozen section to identify histological patterns in stage I lung 
adenocarcinoma of $\leq 3 \mathrm{~cm}$ : accuracy and interobserver agreement. Histopathology. 2015;66:922-38.

17. Nitadori J-i, Bograd AJ, Kadota K, Sima CS, Rizk NP, Morales EA, et al. Impact of micropapillary histologic subtype in selecting limited resection vs lobectomy for lung adenocarcinoma of 2cm or smaller. J Natl Cancer Inst. 2013;105: 1212-20.

18. Cha MJ, Lee HY, Lee KS, Jeong JY, Han J, Shim YM, et al. Micropapillary and solid subtypes of invasive lung adenocarcinoma: clinical predictors of histopathology and outcome. J Thorac Cardiovasc Surg. 2014;147:921-8.e922.

19. Adusumilli PS. Spread through alveolar spaces: an aerogenous invasion in pulmonary adenocarcinomas. J Thorac Cardiovasc Surg. 2016;152:73-4.

20. Neri S, Yoshida J, Ishii G, Matsumura Y, Aokage K, Hishida T, et al. Prognostic impact of microscopic vessel invasion and visceral pleural invasion in non-small cell lung cancer: a retrospective analysis of 2657 patients. Ann Surg. 2014;260: 383-8.

21. Kadota K, Sima CS, Arcila ME, Hedvat C, Kris MG, Jones DR, et al. KRAS mutation is a significant prognostic factor in early-stage lung adenocarcinoma. Am J Surg Pathol. 2016;40:1579-90.

22. Leeman JE, Rimner A, Montecalvo J, Hsu M, Zhang Z, von Reibnitz D, et al. Histologic subtype in core lung biopsies of early-stage lung adenocarcinoma is a prognostic factor for treatment response and failure patterns after stereotactic body radiation therapy. Int J Radiat Oncol Biol Phys. 2017;97:138-45.

23. Travis WD, Brambilla E, Noguchi M, Nicholson AG, Geisinger K, Yatabe Y, et al. Diagnosis of lung cancer in small biopsies and cytology: implications of the 2011 International Association for the Study of Lung Cancer/American Thoracic Society/European Respiratory Society classification. Arch Pathol Lab Med. 2013;137:668-84.

24. Fernandez FG, Kozower BD, Crabtree TD, Force SD, Lau C, Pickens A, et al Utility of mediastinoscopy in clinical stage I lung cancers at risk for occult mediastinal nodal metastases. J Thorac Cardiovasc Surg. 2015;149:35-41. 42.e31.

25. Yeh YC, Kadota K, Nitadori J, Sima CS, Rizk NP, Jones DR, et al. International Association for the Study of Lung Cancer/American Thoracic Society/European Respiratory Society classification predicts occult lymph node metastasis in clinically mediastinal node-negative lung adenocarcinoma. Eur J Cardiothorac Surg. 2016;49:e9-15

26. Donington J, Ferguson M, Mazzone P, Handy J Jr, Schuchert M, Fernando H, et al. American College of Chest Physicians and Society of Thoracic Surgeons consensus statement for evaluation and management for high-risk patients with stage I non-small cell lung cancer. Chest. 2012;142:1620-35.
27. Shirvani SM, Jiang J, Chang JY, Welsh JW, Gomez DR, Swisher S, et al. Comparative effectiveness of 5 treatment strategies for early-stage non-small cell lung cancer in the elderly. Int J Radiat Oncol Biol Phys. 2012;84:1060-70.

28. Ginsberg RJ, Rubinstein LV. Randomized trial of lobectomy versus limited resection for T1 N0 non-small cell lung cancer. Lung Cancer Study Group. Ann Thorac Surg. 1995;60:615-22; discussion 622-3.

29. Yano M, Yoshida J, Koike T, Kameyama K, Shimamoto A, Nishio W, et al. Survival of 1737 lobectomy-tolerable patients who underwent limited resection for cStage IA non-small-cell lung cancer. Eur J Cardiothorac Surg. 2015;47: $135-42$.

30. Nitadori J, Bograd AJ, Morales EA, Rizk NP, Dunphy MP, Sima CS, et al. Preoperative consolidation-to-tumor ratio and SUVmax stratify the risk of recurrence in patients undergoing limited resection for lung adenocarcinoma $\leq 2 \mathrm{~cm}$. Ann Surg Oncol. 2013;20:4282-8.

31. Nakamura H, Saji H, Shinmyo T, Tagaya R, Kurimoto N, Koizumi H, et al. Close association of IASLC/ATS/ERS lung adenocarcinoma subtypes with glucoseuptake in positron emission tomography. Lung Cancer. 2015;87:28-33.

32. Ferretti GR, Busser B, de Fraipont F, Reymond E, McLeer-Florin A, MescamMancini L, et al. Adequacy of CT-guided biopsies with histomolecular subtyping of pulmonary adenocarcinomas: influence of ATS/ERS/IASLC guidelines. Lung Cancer. 2013;82:69-75.

33. Kadota K, Nitadori J, Sima CS, Ujiie H, Rizk NP, Jones DR, et al. Tumor spread through air spaces is an important pattern of invasion and impacts the frequency and location of recurrences after limited resection for small stage I lung adenocarcinomas. J Thorac Oncol. 2015;10:806-14.

34. Lu S, Tan KS, Kadota K, Eguchi T, Bains S, Rekhtman N, et al. Spread through air spaces (STAS) is an independent predictor of recurrence and lung cancerspecific death in squamous cell carcinoma. J Thorac Oncol. 2017;12:223-34.

35. Kadota K, Suzuki K, Kachala SS, Zabor EC, Sima CS, Moreira AL, et al. A grading system combining architectural features and mitotic count predicts recurrence in stage I lung adenocarcinoma. Mod Pathol. 2012;25:1117-27.

36. Eguchi T, Kadota K, Chaft J, Evans B, Kidd J, See Tan K, et al. Cell cycle progression score is a marker for five-year lung cancer-specific mortality risk in patients with resected stage I lung adenocarcinoma. Oncotarget. 2016;7: $35241-56$.

37. Suzuki K, Kadota K, Sima CS, Nitadori J, Rusch VW, Travis WD, et al. Clinical impact of immune microenvironment in stage I lung adenocarcinoma: tumor interleukin-12 receptor beta2 (IL-12Rbeta2), IL-7R, and stromal FoxP3/CD3 ratio are independent predictors of recurrence. J Clin Oncol. 2013;31:490-8. 